\title{
White Noise Disturbance and Topological Heterogeneity Analysis for the Hybrid Consensus Protocol
}

\author{
Haopeng Zhang, Sean R. Mullen, and Qing Hui
}

\begin{abstract}
In this note, we extend the robustness analysis for the hybrid consensus protocol to a more general case: the white noise disturbance effect on the hybrid system, which is a significant measurement when considering the robustness of control protocols. The expectation and covariance of the states for the system are formulated, and the Lyapunov stability for the hybrid system is developed. Furthermore, considering attacks to the graph of the multi-agent system, the topology of the network might be unconnected, therefore, the study of the disconnectedness effect on the system is necessary. Hence, the unconnected topologies are investigated for the hybrid system, and the necessary and sufficient conditions for weak formation and consensus are provided, respectively. Moreover, simulations are provided to verify our theoretical analysis.
\end{abstract}

\section{INTRODUCTION}

The consensus problem for multi-agent systems has significant applications in military and civil arenas including sensor-networked systems, mobile ground vehicle systems, and autonomous underwater vehicles (AUVs), which have recently received a lot of attention [1]-[9]. A general survey of the consensus problem is presented in [3], and the linear system is well discussed. Moreover, a nonlinear consensus protocol, based on system thermodynamic theory, is developed in [4], and the finite-time property and semistability are investigated as well. Both the first order system and the double integrator system are examined. In [2], based on Newtonian mechanics, the velocity and displacement of each agent achieve consensus as time approaches infinity under the double integrator consensus protocol. In addition, hybrid theory is employed to address the consensus problem in [10], which develops a novel framework for solving the fast consensus problem, and also develops semistability for the hybrid consensus system as well.

Besides consensus protocol design, various non-ideal effects to the system have been widely discussed, such as time delay [11], time-varying effects [12], [13], noise disturbance [14] and quantization problems [6], [7], [15]-[17] for information interaction. While considering the attack to the system, the topology of the system might be switching [13], [18], stochastic [5]-[7], [9], or unconnected [19]. In [14], some particular noise disturbances are investigated, and a hybrid formation control protocol is developed. Exact consensus and near consensus are achieved for the continuous and discrete system in [15], [16]. The gossip algorithms

This work was supported by the Defense Threat Reduction Agency, Basic Research Award \#HDTRA1-10-1-0090, to Texas Tech University.

The authors are with the Department of Mechanical Engineering, Texas Tech University, Lubbock, TX 79409-1021, USA (haopeng.zhang@ttu.edu; sean.mullen@ttu.edu; qing.huiettu.edu). that address the random topology consensus problem are introduced in [5]. Furthermore, the quantization of the gossip algorithms is studied and the convergence time and effect of the quantization are developed in [6], [7], [17]. In addition to gossip algorithms, Ergodic theory is employed to solve the random consensus problem [9]. [19] analyzed the heterogeneous networks for double integrator dynamics, showed that the topologies for position and velocity may not be the same or connected, and proposed the necessary and sufficient condition for consensus.

In this paper, the hybrid consensus protocol proposed in [10] is discussed, we extend the result of [14] from some particular noise disturbances to include white noise, since the white noise disturbance effect on the system is a significant observation when considering the robustness of the control protocols. The contributions of this paper are summarized as follows. First, the expectation and covariance matrices are formulated for the continuous system, and, based on Lyapunov stability theory, the stability of the hybrid consensus protocol is developed. Furthermore, the connectivity of the topologies for the hybrid system are investigated. In [19], the authors ignore one case of the eigenvalues, which is the one lying on the imaginary axis (a counterexample is provided). The reason why this case is important is that if the eigenvalue of a linear system lies on the imaginary axis, then the system would not have asymptotic properties. Therefore, in this paper, we develop a sufficient condition for the case where the system has no imaginary eigenvalues. In addition, the necessary and sufficient conditions for weak formation and consensus of the hybrid system are presented, respectively.

The organization of the paper is as follows: the mathematical preliminaries and the hybrid consensus protocol are reviewed in Section II, the main results are presented in Section III, the effects of white noise are provided, and then the connectivity of the topologies is investigated. In Section $\mathrm{V}$, the simulations are provided to illustrate the validity of the developed results. Finally, Section VI concludes the paper.

\section{MAthematicAl PREliminaries}

Firstly, some definitions are introduced. Consider the linear system given by

$$
\dot{x}(t)=A x(t)
$$

Definition 2.1: For $A \in \mathbb{F}^{n \times n}$, and $\lambda \in \operatorname{spec}(\mathrm{A})$, then $\lambda$ is simple if $\operatorname{amult}_{\mathrm{A}}(\lambda)=1$, where amult $_{\mathrm{A}}$ denotes the algebraic multiplicity of an eigenvalue $\lambda$ of $A$. 
Definition 2.2: For $A \in \mathbb{R}^{n \times n}$, A is semistable if $\operatorname{spec}(\mathrm{A}) \subset \mathrm{CLHP}$, and if $0 \in \operatorname{spec}(\mathrm{A})$, then 0 is semisimple, where CLHP stands for closed left half plane in $\mathbb{C}$.

Define $E_{A}$ as the eigenvector space for $A$. To efficiently describe a networked environment for a control system, graph theory is a powerful tool. In this paper, we use graph-related notation to describe our network model. More specifically, let $\mathscr{G}=(\mathscr{V}, \mathscr{E}, \mathscr{A})$ denote an undirected graph with the set of vertices $\mathscr{V} \doteq\left\{v_{1}, v_{2}, v_{3}, \ldots\right\}$ and $\mathscr{E} \subseteq \mathscr{V} \times \mathscr{V}$ represents the set of edges. The matrix $\mathscr{A}$ with nonnegative adjacency elements $a_{i, j}$ serves as the weighted adjacency matrix. The node index of $\mathscr{G}$ is denoted by a finite index set $\mathscr{N}=\{1,2,3, \ldots\}$. An edge of $\mathscr{G}$ is denoted by $e_{i, j}=$ $\left(v_{i}, v_{j}\right)$ and the adjacency elements associated with the edges are positive. We assume $e_{i, j} \in \mathscr{E} \Leftrightarrow a_{i, j}=1$ and $a_{i, i}=0$ for all $i \in \mathscr{N}$. If there is a path from any node to any other node in the graph, then we call the graph connected.

The hybrid consensus protocol via homogeneous topology $G$ we consider in this paper is given by

$$
\begin{aligned}
& \dot{x}_{c i}(t)=-\sum_{j=1, j \neq i}^{q} C_{i, j}^{G}\left(x_{c i}(t)-x_{c j}(t)\right) \\
& -\sum_{j=1, j \neq i}^{q} C_{i, j}^{G}\left(x_{i}(t)-x_{j}(t)-w_{i, j}\right) \\
& \left(x_{i}(t), \bar{x}_{i}(t), x_{c i}(t), \bar{x}_{c i}(t)\right) \notin Z_{i} \\
& \begin{array}{c}
x_{c i}(0)=x_{c i 0}, \quad t \geq 0 \\
\dot{x}_{i}(t)=\sum_{j=1, j \neq i}^{q} C_{i, j}^{G}\left(x_{c i}(t)-x_{c j}(t)\right)
\end{array} \\
& x_{c i}\left(t^{+}\right)=\operatorname{argmin}_{x_{c i}(t)} \sum_{j=1, j \neq i}^{q} C_{i, j}^{G}\left\|x_{c i}(t)-x_{c j}(t)\right\|_{2}^{2} \\
& \left(x_{i}(t), \bar{x}_{i}(t), x_{c i}(t), \bar{x}_{c i}(t)\right) \in Z_{i}
\end{aligned}
$$

where the resetting set $Z_{i}$ is given by

$$
\begin{array}{r}
Z_{i}=\left\{\left(x_{i}, \bar{x}_{i}, x_{c i}, \bar{x}_{c i}\right): \frac{d}{d t} L_{i}\left(x_{i}, \bar{x}_{i}\right)=0\right. \\
\left.L_{i}\left(x_{c i}, \bar{x}_{c i}\right)>\min _{x_{c i}} L_{i}\left(x_{c i}, \bar{x}_{c i}\right)\right\}
\end{array}
$$

or

$$
\begin{array}{r}
Z_{i}=\left\{\left(x_{i}, \bar{x}_{i}, x_{c i}, \bar{x}_{c i}\right): \frac{d}{d t} L_{i}\left(x_{c i}, \bar{x}_{c i}\right)=0\right. \\
\left.L_{i}\left(x_{c i}, \bar{x}_{c i}\right)>\min _{x_{i}} L_{i}\left(x_{c i}, \bar{x}_{c i}\right)\right\}
\end{array}
$$

where $L_{i}\left(x_{i}, \bar{x}_{i}\right)=\sum_{j=1, j \neq i}^{q} C_{i, j}\left\|x_{i}(t)-x_{j}(t)\right\|_{2}^{2}$ and $L_{i}\left(x_{c i}, \bar{x}_{c i}\right)=\sum_{j=1, j \neq i}^{q} C_{i, j}\left\|x_{c i}(t)-x_{c j}(t)\right\|_{2}^{2}$. Finally, the matrix $C$ is a connectivity matrix for the graph $\mathscr{G}$ defined by $C_{i, j}^{G}=0$ if $(i, j) \notin \mathscr{E}, C_{i, j}^{G}=1$ if $(i, j) \in \mathscr{E}$, and $C_{i, i}^{G}=-\sum_{k=1, k \neq i}^{q} C_{i, k}, i \neq j, i, j=1, \ldots, q$.

This hybrid protocol is a state-dependent, dynamic hybrid consensus protocol proposed in [10], which can be viewed as an observer-based controller design. We call $x_{c i}$ the observer state and $x_{i}$ the plant state. Then the hybrid consensus protocol (2) describes a feedback interconnection between the plant and observer. The motivation of this hybrid consensus protocol comes from designing fast convergent consensus protocols for multi-agent coordination via output feedback using dynamic compensators. We use the idea of thermodynamic stabilization with impulsive control [20] to design such a controller. In this case, the resetting set $Z_{i}$ is defined to be the set of all points in the closed-loop state space that correspond to decreasing the Laplacian disagreement function $L_{i}\left(x_{i}, \bar{x}_{i}\right)$ [21] of the $i$ th subsystem and minimizing the Laplacian disagreement function $L_{i}\left(x_{c i}, \bar{x}_{c i}\right)$ of the $i$ th agent. Note that the expression of $x_{c i}\left(t^{+}\right)$is equivalent to a least squares minimization problem which has the solution

$$
\begin{aligned}
& x_{c i}\left(t^{+}\right)=\frac{1}{\left|\mathcal{K}_{i}\right|} \sum_{j \in \mathcal{K}_{i}} x_{c j}(t), \\
& \left(x_{i}(t), \bar{x}_{i}(t), x_{c i}(t), \bar{x}_{c i}(t)\right) \in Z_{i},
\end{aligned}
$$

where $\mathcal{K}_{i}$ denotes the indices of all the other agents which have a communication link with the $i$ th agent and $\left|\mathcal{K}_{i}\right|$ denotes the cardinality of the set $\mathcal{K}_{i}$. Equation (5) is similar in form to the model proposed in [22]. Furthermore, (5) implies that the resetting dynamics are such that the state of the controller is reset to the center of gravity or average value of all other controller state variables. More detailed explanation of (2), (3), and (4) can be found in [10]. Under these consensus protocols, it is shown that the network achieves consensus quite rapidly and even in finite time [10].

\section{MAIN RESULT}

In this section, we intend to investigate the robustness of the hybrid consensus protocol in Section II with the presence of white noise.

\section{A. Robustness of the continuous consensus protocol}

The continuous-time part of the protocol (2) can be represented in a vector form. Defining $X=$ $\left[\begin{array}{llllll}x_{1} & \cdots & x_{q} & x_{c 1} & \cdots & x_{c q}\end{array}\right]^{\mathrm{T}}$, then the continuous-time system becomes

$$
\dot{X}=\Phi X+\Psi
$$

where

$$
\Psi=\left[\begin{array}{c}
0 \\
\vdots \\
0 \\
-\sum_{j \in N_{1}} a_{1, j} w_{1, j} \\
-\sum_{j \in N_{2}} a_{2, j} w_{2, j} \\
\vdots \\
-\sum_{j \in N_{q}} a_{q, j} w_{q, j}
\end{array}\right]
$$

$\Phi=\left[\begin{array}{cc}0 & 1 \\ -1 & -1\end{array}\right] \otimes L$, and $L$ is the Laplacian matrix for the graph topology of the networked system.

We firstly consider a more general case. Consider the following system

$$
\dot{X}=\Phi X+B \mathrm{w}
$$

where $\Phi \in \mathbb{R}^{n \times n}$ is the state matrix, $B \in \mathbb{R}^{n \times n}$ is the weight matrix, and $w \in \mathbb{R}^{n \times 1}$ is the vector of the standard Gaussian 
white noise process. To characterize the system,we definite expectation of the states as $\mathbb{E}(X)$ and covariance of $x(t)$ by $Q(t) \triangleq \mathbb{E}\left[x(t) x(t)^{\mathrm{T}}\right]$ and we then present our main result about the system (7).

Theorem 3.1: Consider the system (7) with white noise disturbance, and assume that $\mathbb{E}(X(0))$ is given. Then, the expectation and covariance of the states are

$$
\begin{aligned}
\mathbb{E}(X(t)) & =\exp \Phi t \times \mathbb{E}\left(X_{0}\right), \\
Q(t) & =e^{\Phi t} Q(0) e^{\Phi^{\mathrm{T}} t}+\int_{o}^{t} e^{\Phi s} B B^{\mathrm{T}} e^{\Phi^{\mathrm{T}} s} \mathrm{ds}
\end{aligned}
$$

The following question is whether the covariance matrix is finite or infinite, which would thus effect the robustness of our hybrid consensus protocol. Therefore, the following proposition presents necessary and sufficient conditions for well-defined covariance matrix $Q(t)$.

Proposition 3.1: Consider the system (7), and assume $\Phi$ is semistable. Then $\int_{o}^{t} e^{\Phi s} B B^{\mathrm{T}} e^{\Phi^{\mathrm{T}} s} \mathrm{ds}<\infty$ if and only if $\operatorname{ker} \Phi \subset \operatorname{ker} B$.

Next, we narrow the scope of the general result of Theorem 3.1 into our consensus case.

Corollary 3.1: Consider the system (6). If $w_{i, j}$ is the Gaussian white noise process, then the explicit formulations of the expectation and covariance are

$$
\begin{aligned}
\mathbb{E}(X(t))= & e^{\left[\begin{array}{cc}
0 & 1 \\
-1 & -1
\end{array}\right] \otimes L t} \times \mathbb{E}\left(X_{0}\right) \\
Q(t)= & e^{\Phi t} Q(0) e^{\Phi^{\mathrm{T}} t} \\
& +\int_{o}^{t} e^{\Phi s}\left[\begin{array}{ll}
0 & 0 \\
0 & 1
\end{array}\right] \otimes L L^{\mathrm{T}} e^{\Phi^{\mathrm{T}} s} \mathrm{ds}
\end{aligned}
$$

Furthermore, as $t \rightarrow \infty$,

$$
\begin{aligned}
& \mathbb{E}(\infty)=\left[\begin{array}{cc}
\frac{1}{q} \times \mathbf{1 1}^{\mathrm{T}} & 0 \\
0 & \frac{1}{q} \times \mathbf{1 1}^{\mathrm{T}}
\end{array}\right] \mathbb{E}\left(X_{0}\right) \\
& Q(\infty)=\mathbf{C}
\end{aligned}
$$

where $\mathbf{C}$ is a constant matrix.

\section{B. Robustness of the hybrid consensus protocol}

In this subsection, we investigate the jump process's effect on the continuous-time linear system (6).

Theorem 3.2: Under the hybrid consensus protocol (2), the system's expectation is Lyapunov stable under white noise disturbances.

\section{Disconnected Topology AnAlysis}

The connectivity of the topology has a significant impact on the system, so in this section, we explore the disconnected topologies' influence on the system.

\section{A. Continuous subsystem of the hybrid protocol}

The problem we consider here is

$$
\dot{X}=\left[\begin{array}{cc}
\mathbf{0} & L_{1} \\
-L_{2} & -L_{3}
\end{array}\right] X
$$

$L_{1}, L_{2}$, and $L_{3}$ are the Laplacian matrices for the topologies $G_{1}, G_{2}$, and $G_{3}$, which are the control observer topology, plant topology, and observer topology, respectively. A question that may be raised in relation to the three topologies is if we should have to guarantee that all topologies of our protocol are connected to achieve consensus for the plant. Are there any looser assumptions that can be made about the connectivity of the topologies? Therefore, in this subsection, we study the connectivity of those three topologies, and discuss two significant cases.

Firstly, let $\hat{\Phi}=\left[\begin{array}{cc}\mathbf{0} & L_{1} \\ -L_{2} & -L_{3}\end{array}\right]$. Next, we will investigate the properties of the matrix $\hat{\Phi}$.

$$
\operatorname{det}\left(\hat{\Phi}-s I_{2 q \times 2 q}\right)=\operatorname{det}\left(s^{2} I_{q \times q}+L_{3} s+L_{1} L_{2}\right)
$$

and define $P(s)=s^{2} I_{q \times q}+s L_{3}+L_{1} L_{2}$. The following lemma presents the relation between the $\hat{\Phi}$ matrix and $P(s)$ matrix.

Lemma 4.1: The eigenvalues of the matrix $\hat{\Phi}$ are the solution to the following equation:

$$
v_{p}^{\mathrm{T}} p(s) v_{p}=0
$$

where $v_{p}$ is the eigenvector of the matrix $P(s)$ corresponding to eigenvalue zero, and $v_{p}^{\mathrm{T}} v_{p}=1$.

Remark 4.1: In [19], the authors mentioned that $P(s)$ and $L$ have the same spectra, but it is relatively straightforward to check that they are not equal to each other. The relation between those two matrices is that the eigenvalues of $\hat{\Phi}$ are the solution to the following equation:

$$
v_{p}^{\mathrm{T}} p(s) v_{p}=0
$$

where $v_{p}$ is the eigenvector of the matrix $P(s)=s^{2} I_{q \times q}+$ $s L_{\dot{x}}+L_{x}$ corresponding to eigenvalue zero, and $v_{p}^{\mathrm{T}} v_{p}=1$.

Next, the Jordan form of matrix $\hat{\Phi}$ is presented.

Lemma 4.2: Consider the matrix $\hat{\Phi}$. If there exist $m v_{p}$ satisfying $v_{p} \in\left\{\operatorname{ker}\left(L_{3}\right) \cap E_{L_{1} L_{2}} \backslash \operatorname{ker}\left(L_{1} L_{2}\right)\right\}$, then

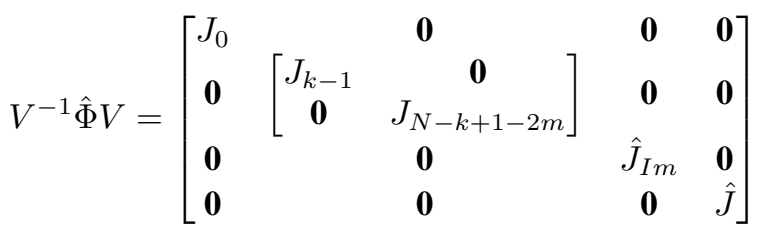

where $J_{0}=\left[\begin{array}{ll}0 & 0 \\ 0 & 0\end{array}\right], J_{k-1}=\operatorname{diag}\left\{0_{1}, 0_{2}, \cdots, 0_{k-1}\right\}$, $J_{N-k+1-2 m}=\operatorname{diag}\left\{0_{1}, 0_{2}, \cdots, 0_{N-k+1-2 m}\right\}$ $\hat{J}_{I m}=\operatorname{diag}\left(b_{1 i},-b_{1 i}, \cdots, b_{m i},-b_{m i}\right), \quad \hat{J}=$ $\operatorname{diag}\left\{a_{1}, a_{2}, \cdots, a_{2 q-2-N}\right\}, k$ is the number of components of $G_{2}, a_{i}<0, N$ is the dimension of $\operatorname{ker} L_{1} L_{2}$ (i.e., $\left.\operatorname{dim}\left(\operatorname{ker} L_{1} L_{2}\right)=N\right)$, and $m$ is the number of pairs of the imaginary eigenvalues of matrix $\hat{\Phi}$.

After the discussion of the eigenvalues of $\hat{\Phi}$, the results of the eigenvectors are presented.

Lemma 4.3: For matrix $\hat{\Phi}, V(1)=\frac{1}{q}\left[\begin{array}{ll}\mathbf{1}^{\mathrm{T}} & \mathbf{0}^{\mathrm{T}}\end{array}\right]^{\mathrm{T}}$ and $V(2)=\frac{1}{q}\left[\begin{array}{ll}\mathbf{0}^{\mathrm{T}} & \mathbf{1}^{\mathrm{T}}\end{array}\right]^{\mathrm{T}}$ are the eigenvectors corresponding to $s_{1}=s_{2}=0$, respectively. $V(i)=\frac{1}{p_{i}}\left[\begin{array}{ll}v_{L 2, i}^{\mathrm{T}} & \mathbf{0}^{\mathrm{T}}\end{array}\right]^{\mathrm{T}}$ where $p_{i}=k_{i-1}$ are the eigenvectors corresponding to $J_{k-2 m}, i=3, \cdots, N-2 m, v_{L 2, i}$ is the eigenvector for 
$L_{2}$ corresponding to 0 , which is defined in Corollary 1 of [19].

To avoid having eigenvalues lie on the imaginary axis for matrix $\hat{\Phi}$, the sufficient condition is developed.

Lemma 4.4: For matrix $\hat{\Phi}$, if $G_{3}$ is a connected graph, then there are no eigenvalues lying on the imaginary axis.

The Jordan form of $\hat{\Phi}$ is presented in the following lemma for connected graph $G_{3}$

Lemma 4.5: Consider the matrix $\hat{\Phi}$, assume $G_{3}$ is connected, then

$$
V^{-1} \hat{\Phi} V=\left[\begin{array}{ccc}
J_{0} & \mathbf{0} & \mathbf{0} \\
\mathbf{0} & J_{N-1} & \mathbf{0} \\
\mathbf{0} & \mathbf{0} & \hat{J}
\end{array}\right]
$$

where $J_{0}=\left[\begin{array}{ll}0 & 0 \\ 0 & 0\end{array}\right], J_{N-1}=\operatorname{diag}\left\{0_{1}, 0_{2}, \cdots, 0_{N-1}\right\}$, $\hat{J}=\operatorname{diag}\left\{a_{1}, a_{2}, \cdots, a_{2 q+1-N}\right\}, \mathrm{k}$ is the number of the components of $G_{2}$, and $a_{i}<0$, and $N=\operatorname{dim}\left(\operatorname{ker} L_{1} L_{2}\right)$.

Two important cases are discussed in the following, and the weak formation and consensus could be achieved due to some looser assumptions of connectivity regarding the plant graph and observer graph.

1) Weak formation problem: Due to possible link failures and link creations of communications between agents, the agents may connect or disconnect with each other via wireless communications during the movement. Hence, the plant topology of the multi-agent system may not be connected at all times. However, we can guarantee a connected observer topology since it is designed by ourselves. So the question is: Can we achieve consensus for the multi-agent system in this case? The answer, unfortunately, is no. Besides this negative answer, we also want to know what this scenario really looks like in order to remedy our design. This leads to the following result regarding weak formation.

Theorem 4.1: Considering the system (12), assume $G_{1}$ and $G_{2}$ are disconnected, and that $G_{3}$ is connected. It then follows that the system becomes

$$
\begin{aligned}
X(\infty)= & \exp \hat{\Phi}(\infty)\left[\begin{array}{c}
X(0) \\
X_{c}(0)
\end{array}\right] \\
= & {\left[\begin{array}{cc}
\frac{1}{q} \mathbf{1 1}^{\mathrm{T}}+\sum_{2}^{k} v_{\left(L_{2}\right), i} * v_{\left(L_{2}\right), i}^{\mathrm{T}} & \mathbf{0}^{q \times q} \\
\mathbf{0}^{q \times q} & \frac{1}{q} \mathbf{1 1}^{\mathrm{T}}
\end{array}\right] } \\
& +\sum_{j=2 q-k-1}^{p} V(j) V^{-1}(j)\left[\begin{array}{c}
X(0) \\
X_{c}(0)
\end{array}\right]
\end{aligned}
$$

where $p=\operatorname{dim}\left(\operatorname{ker} L_{1} L_{2}\right)-\operatorname{dim}\left(\operatorname{ker} L_{2}\right)$

To achieve consensus for the observer states, we develop the result of Theorem 4.1. Before stating the result, the following lemma is needed:

Lemma 4.6: If graph $G_{1}$ is connected, and graph $G_{2}$ is disconnected, then $\operatorname{ker} L_{2}=\operatorname{ker} L_{1} L_{2}$.

Theorem 4.2: Considering the system (12), and assuming $G_{3}$ and $G_{1}$ are connected, $G_{2} \neq \mathbf{0}_{n \times n}$ are disconnected.
Then, as $t$ approaches infinity, the system becomes

$$
\begin{aligned}
& X(\infty)=\exp \hat{\Phi}(\infty)\left[\begin{array}{c}
X(0) \\
X_{c}(0)
\end{array}\right] \\
& =\left[\begin{array}{cc}
\frac{1}{q} \mathbf{1 1}^{\mathrm{T}}+\sum_{2}^{k} v_{L 2, i} * v_{L 2, i}^{\mathrm{T}} & \mathbf{0}^{q \times q} \\
\mathbf{0}^{q \times q} & \frac{1}{q} \mathbf{1 1}^{\mathrm{T}}
\end{array}\right]\left[\begin{array}{c}
X(0) \\
X_{c}(0)
\end{array}\right](19)
\end{aligned}
$$

Remark 4.2: The formation achieved here is determined by the disconnected topology $G_{2}$, and we cannot adjust this formation, so it is called weak formation. However, in [14], the constant noise disturbance can achieve arbitrary formation for the hybrid system, therefore, we might achieve arbitrary formation if we add a constant term into the disconnected system.

2) Consensus problem: The weak formation control for the networked system can not satisfy our consensus aim, therefore, we assume the plant topology is connected. So, the question is, can we use the limited observer to achieve consensus?

Theorem 4.3: Consider the system (12). Assuming $G_{1}$ and $G_{2}$ are connected and not necessarily the same, $G_{3}$ is disconnected, and the system achieves consensus as $t$ goes to infinity if and only if $v_{p} \notin\left\{\operatorname{ker}\left(L_{3}\right) \cap E_{L_{1} L_{2}} \backslash \operatorname{ker}\left(L_{1} L_{2}\right)\right\}$

$$
\begin{aligned}
X(\infty) & =\exp \hat{\Phi}(\infty)\left[\begin{array}{c}
X(0) \\
X_{c}(0)
\end{array}\right] \\
& =\left[\begin{array}{ll}
\frac{1}{q} \mathbf{1 1}^{\mathrm{T}} & \mathbf{0}^{q \times q} \\
\mathbf{0}^{q \times q} & \frac{1}{q} \mathbf{1 1}^{\mathrm{T}}
\end{array}\right] \times\left[\begin{array}{c}
X(0) \\
X_{c}(0)
\end{array}\right]
\end{aligned}
$$

B. Lyapunov stability for disconnected hybrid network systems

The continuous subsystem of the disconnected hybrid network system has been investigated in Subsections IV-A.1 and IV-A.2. In this subsection, the Lyapunov stability for the whole disconnected hybrid protocol is discussed.

Theorem 4.4: Under the hybrid consensus protocol (2), the system is Lyapunov stable via disconnected topologies $G_{1}, G_{2}$, and connected topology $G_{3}$.

\section{Simulations}

In this section, Matlab is employed to verify our theoretical analysis for the hybrid consensus protocol. The connected homogeneous hybrid system in ideal condition is shown as Fig. 1, and the white noise disturbance to the connected homogeneous hybrid system is shown as Fig. 2. Weak formation can be observed in Fig. 3, and observer consensus was achieved in Fig. 4. In a particular case, the eigenvalue lying on the imaginary axis is shown in Fig. 6. A counterexample of [19] is provided in Fig. 7.

\section{CONCLUSION}

In this paper, the robustness of the hybrid consensus protocol is investigated for a more general noise disturbancewhite noise. The expectation and covariance matrices for the system are formulated, and the Lyapunov stability theory for the hybrid system is developed. Moreover, the connectivity of the graph is found to play a significant role in the analysis of the consensus protocol, and sufficient condition for the 


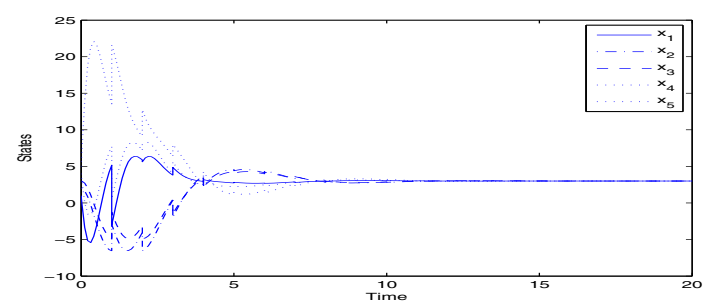

(a) Plant states

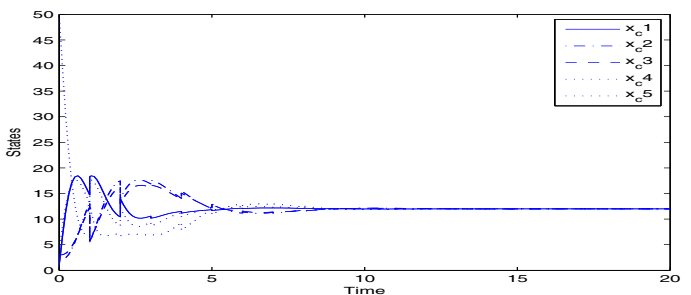

(b) Observer states

Fig. 1. Connected homogeneous topology for the system under ideal condition

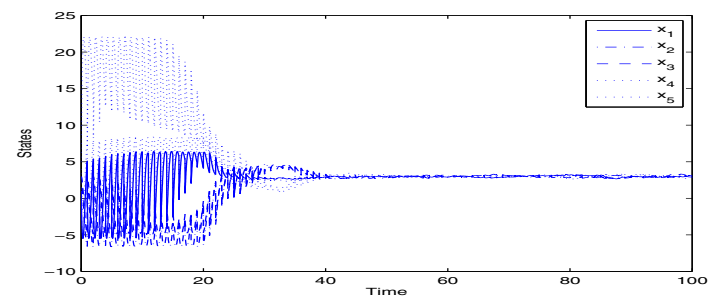

(a) Plant states

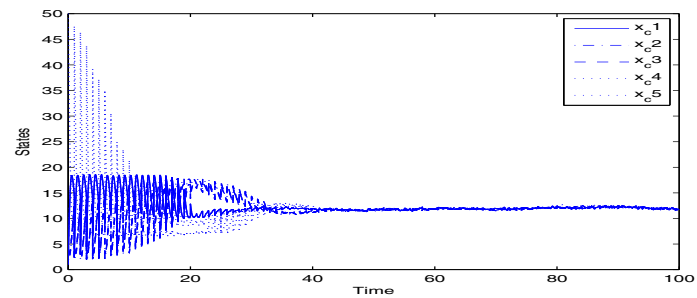

(b) Observer states

Fig. 2. Connected topologies for the system with white noise disturbance

disconnected system having asymptotic property is proposed. Weak formation and consensus could be achieved via disconnected graphs, thus, under some conditions, the hybrid consensus protocol can achieve consensus via heterogeneous topologies.

\section{REFERENCES}

[1] R. Olfati-Saber, J. A. Fax, and R. M. Murray, "Consensus problems in networks of agents with switching topology and time-delays," Proc. IEEE, vol. 95, pp. 215-233, 2007.

[2] J. Cortes, S. Martinez, and F. Bullo, "Consensus control for a class of networks of dynamic agents," Int. J. Robust Nonlinear Control, vol. 17, pp. 941-959, 2006.

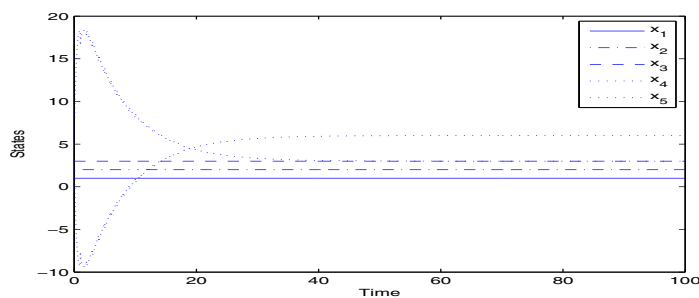

(a) Plant states

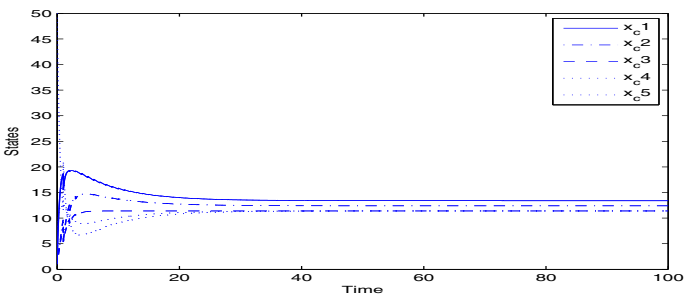

(b) Observer states

Fig. 3. $G_{3}$ is connected; $G_{1}$ and $G_{2}$ are unconnected: weak formation

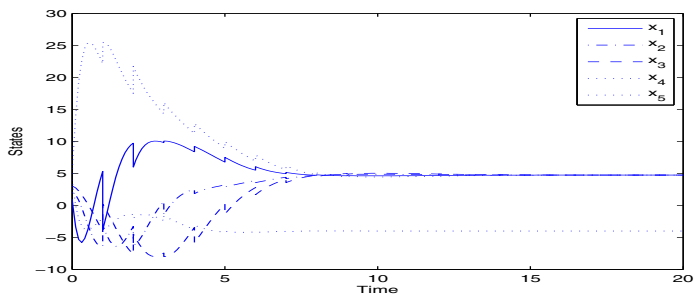

(a) Plant states

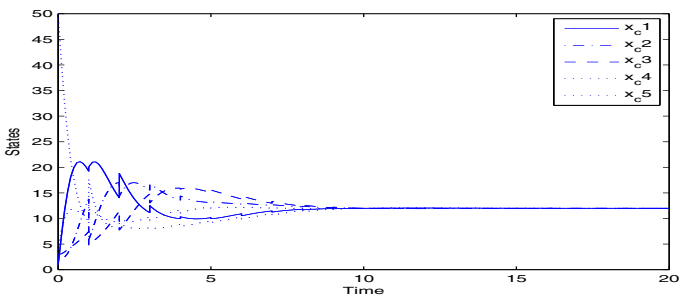

(b) Observer states

Fig. 4. $G_{3}$ is connected; $G_{1}$ and $G_{2}$ are unconnected: observer consensus

[3] W. Ren and R. W. Beard, Distributed Consensus in Multi-vehicle Cooperative Control Theory and Applications. London, U.K.: SpringerVerlag, 2008.

[4] Q. Hui, W. M. Haddad, and S. P. Bhat, "Semistability, finite-time stability, differential inclusions, and discontinuous dynamical systems having a continuum of equilibria," IEEE Trans. Autom. Control, vol. 54, no. 10, pp. 2465-2470, 2009.

[5] S. Bod, A. Ghosh, B. Prabhakar, and D. Shah, "Randomized gossip algorithms," IEEE Trans. Info. Theory, vol. 52, pp. 2508-2530, 2006.

[6] J. Lavaei and R. M. Murray, "On quantized consensus by means of gossip algorithm-part i: Convergence proof," in Proc. Amer. Control Conf., St. Louis, MO, 2009.

[7] —, "On quantized consensus by means of gossip algorithm-part ii: Convergence time," in Proc. Amer. Control Conf., St. Louis, MO, 2009.

[8] A. Tahbaz-Salehi and A. Jadbabaie, "Necessary and sufficient conditions for consensus over random independent and identically dis- 


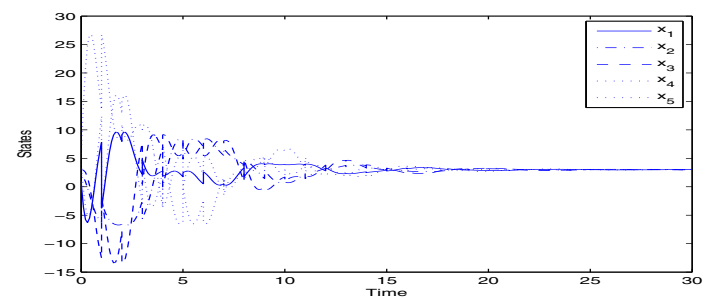

(a) Plant states

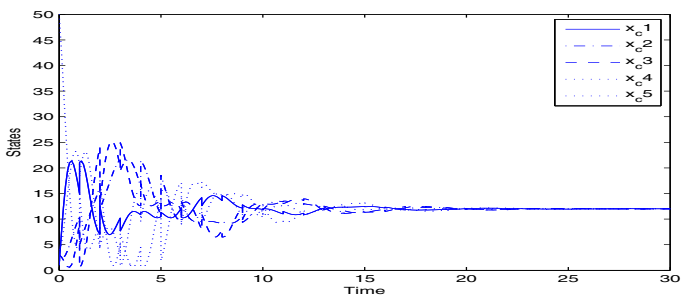

(b) Observer states

Fig. 5. $G_{1}$ and $G_{2}$ are connected; $G_{3}$ is unconnected: consensus

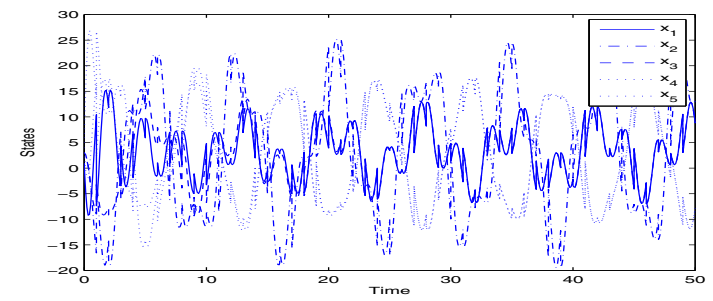

(a) Plant states

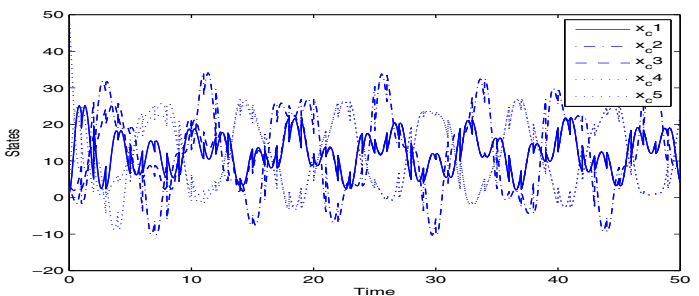

(b) Observer states

Fig. 6. Case of eigenvalues lying on the imaginary axis

tributed switching graphs," in Proc. IEEE Conf. Decision Control, New Orleans, LA, 2007, pp. 791-795.

[9] Q. Hui and H. Zhang, "Ergodicity of flocking systems for infinitedimensional multi-agent coordination," in Proc. IEEE Conf. Decision Control, Atlanta, GA, 2010, pp. 5750-5755.

[10] Q. Hui, "Hybrid consensus protocols: An impulsive dynamical system approach," Int. J. Control, vol. 83, pp. 1107-1116, 2010.

[11] —, "Stability and convergence of nonlinear systems having a continuum of equilibria and time-varying delays," in Proc. IEEE Conf. Decision Control, Atlanta, GA, 2010, pp. 2251-2256.

[12] Q. Hui and W. M. Haddad, "Uniform semistability for time-varying dynamical systems and network consensus with time-dependent communication links," in Proc. Amer. Control Conf., St. Louis, MO, 2009, pp. 4050-4055.

[13] Q. Hui, W. M. Haddad, and S. P. Bhat, "Semistability for time-varying discontinuous dynamical systems with application to agreement problems in switching networks," in Proc. IEEE Conf. Decision Control,

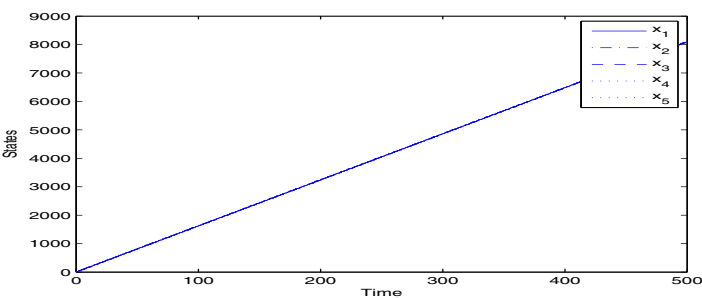

(a) Position

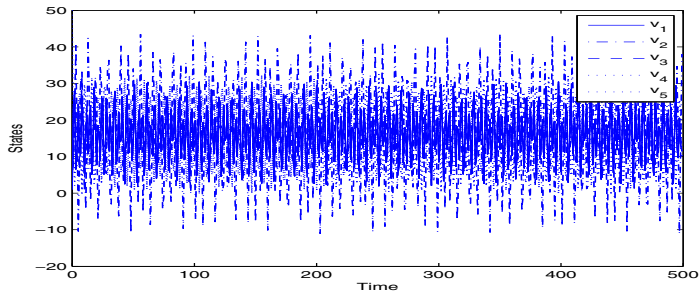

(b) Velocity

Fig. 7. Counterexample

Cancun, Mexico, 2008, pp. 2985-2990.

[14] H. Zhang, S. R. Mullen, and Q. Hui, "A note to robustness analysis of the hybrid consensus protocols," in Proc. Amer. Control Conf., San Francisco, CA, 2011.

[15] Q. Hui, "Quantized near-consensus via quantized communication links," in Proc. Amer. Control Conf., Baltimore, MD, 2010, pp. $247-$ 252.

[16] H. Zhang and Q. Hui, "Distributed consensus under limited information," in 19th Int. Symp. Math. Theory Networks Syst., Budapest, Hungary, 2010, pp. 2253-2257.

[17] R. Carli, P. Frasca, F. Fagnani, and S. Zampieri, "Gossip consensus algorithms via quantized communication," vol. 46, pp. 70-80, 2010.

[18] Q. Hui, "Lyapunov-based semistability analysis for discrete-time switched network systems," in Proc. Amer. Control Conf., San Francisco, CA, 2011.

[19] D. Goldin, S. A. Attia, and J. Raisch, "Consensus for double integrator dynamics in heterogeneous networks," in Proc. IEEE Conf. Decision Control, Atlanta, GA, 2010, pp. 4504-4510.

[20] W. M. Haddad, V. Chellaboina, Q. Hui, and S. G. Nersesov, "Energyand entropy-based stabilization for lossless dynamical systems via hybrid controllers," IEEE Trans. Autom. Control, vol. 52, no. 9, pp 1604-1614, 2007.

[21] R. Olfati-Saber and R. M. Murray, "Consensus problems in networks of agents with switching topology and time-delays," IEEE Trans. Autom. Control, vol. 49, pp. 1520-1533, 2004.

[22] T. Vicsek, A. Czirók, E. Ben-Jacob, I. Cohen, and O. Shochet, "Novel type of phase transition in a system of self-deriven particles," Phys. Rev. Lett., vol. 75, pp. 1226-1229, 1995. 